\title{
The Effect of Perceived Organizational Support on Job Performance Among Administrative Staff of Newly-Built University in China
}

\author{
Peng Wan* \\ School of Education and Modern Languages, University Utara Malaysia, Malaysia \\ Department of Human Resources, Yibin University, China \\ Khaliza Binti Saidin \\ School of Education and Modern Languages, University Utara Malaysia, Malaysia
}

\begin{abstract}
In the context of the popularization of higher education and the emergence of a large number of newly-built universities in China, it is important to improve the job performance of administrative for newly-built university effectiveness and outcomes. According to perceived organizational support (POS) theory and relevant research review, POS is considered to be a key factor in improving job performance. However, there is still some research found that POS could not affect job performance directly and researchers are less concerned about university administrative staff. Therefore, this study aimed to determine the effect of perceived organizational support on job performance among administrative staff of newly-built university in China. An online questionnaire was adopted in the study to collect data, and a total of 426 administrative staff participated in the survey. After data analysis by SPSS, the findings indicated that the level of POS and job performance among administrative staff is slightly low. The findings also revealed that a positive correlation exists between POS and job performance, and the POS has significant effect on job performance. The study further discussed the findings and recommended that more organizational support should be provided by newly-built universities in order to improve the job performance of administrative staff.
\end{abstract}

Keywords: Perceived organizational support; Job performance; Administrative staff; Newly-built University; China.

(a) 1 () CC BY: Creative Commons Attribution License 4.0

\section{Introduction}

The Chinese Higher Education Quality Report (Higher Education Evaluation Center of the Chinese Ministry of Education, 2017) for the first time shows that a large number of new universities have been born in China since 2000. By the end of 2017, there were 1219 universities nationwide, including 678 newly-built universities, accounting for $55.6 \%$ of the total number of Chinese universities. The newly-built universities have become an important part of Chinese higher education institutions, and the improvement of their administration level is of great significance to the improvement of the level of Chinese higher education (Zhang and Xie, 2015). In the education management, job performance of education practitioners is considered of pivotal for university effectiveness and outcomes. How to strengthen the job performance of the newly-built university administrative staff and thus better serve the teaching and research of the university is a major problem that the newly-built university urgently need to solve in the process of construction and development (Zhuang, 2017).

Perceived organizational support (POS) is considered of as a key factor in improving job performance of employees (Rhoades and Eisenberger, 2002). In recent years, with the popularization of the concept and theory of POS in the academia, more and more research has been conducted to study the impact of POS on job performance. Review of literature mostly shows that POS has a positive influence on the several work outcomes that include job satisfaction, work engagement, commitment and job performance (Ahmed and Nawaz, 2015; Rhoades and Eisenberger, 2002). Especially for job performance, there are also lots of empirical research which indicated that the POS has a positive influence on job performance (Afzali et al., 2014; Chen and Yin, 2009; Mohamed and Ali, 2015), whereas some research revealed that POS can not affect job performance significantly, unless it is mediated by other variables, such as organizational behavior (Chiang and Hsieh, 2012). Moreover, some researchers found that the POS can not influence work performance of employees (Labrague et al., 2018). It reveals that the effect of POS on job performance may be different among different sample population in different organizational culture background.

On the other hand, it can be seen that the study of organizational support and job performance has involved business, medical and tourism practitioners, but it has seldom been involved in the education industry. Particularly, researchers are less concerned about university administration. Further empirical research on the relationship between perceived organizational support and job performance in diverse contexts and different target population is needed. In particular, the study on the impact of the POS on the job performance among administrative staff in Chinese newly-built universities was still blank. Therefore, this study was going to fill this academic gap. Taking into account previous study evidence indicating the probably significant relation between POS and job performance, 
this paper took a unique perspective as it attempted to assess the contribution of POS directly on job performance among administrative staff in Chinese newly-built universities.

The concept of POS was proposed firstly by Eisenberger et al. (1986). This concept was proposed in the academic context of previous research that has placed great emphasis on employee commitment to the organization but rarely paid attention to the organization's commitment to employees. To be specific, POS is a comprehensive view of the employees of whether organization value their contributions and caring about their well-being Eisenberger et al. (1986). Based on the theory of social exchange and the norm of reciprocity, Eisenberger et al. (1986), Rhoades and Eisenberger (2002) further developed the theory of POS which believe that POS can increase obligation of employees to help organization to reach its goal, and the outcomes of POS include performance increasing and absenteeism and turnover decreasing. Based on the literature review and the underlying theory, the following study was conducted.

\section{Research Objective}

The research objective was to determine the effect of POS on job performance among administrative staff of newly-built university in China. To be specific, this study focus on:

(i ) Determine the level of POS and job performance among administrative staff.

(ii ) Examine the correlation between POS and job performance among administrative staff.

(iii) Exploring the effect of POS on job performance among administrative staff.

\section{Research Questions}

In order to achieve the research objective, the following questions were proposed:

(i ) What is the level of POS and job performance among administrative staff?

(ii What is the correlation between POS and job performance among administrative staff?

(iii) Does POS have a significant effect on job performance among administrative staff?

\section{Methodology}

This study employed quantitative research design using a survey approach. Moreover, the online questionnaire method was carried out for data collection since it is more convenient and effective (Jing et al., 2008). Total of 426 administrative staff from different newly-built universities responded questionnaires which were distributed by email, QQ, WeChat, and the OA (Office Automation) system of the university in China. The questionnaire was made up of two scales. One is the POS scale constructed by Ling et al. (2006) while the other one is job performance scale developed by Yu (1996). These two scales are widely used in China, which is more suitable for Chinese cultural background, and their reliability and validity have been tested many times. In addition, a five points Likert scale was employed for the items in the questionnaire.

The data collected in the study were analyzed using SPSS version 25. The values of the reliability of POS scale and job performance scale are 0.947 and 0.976 respectively, which means that the reliability of questionnaire is excellent. In addition, the construct validity was also confirmed by exploratory factor analysis (EFA) with all items factor loading values ranging from 0.672 to 0.863 .

\section{Results}

Research Question 1: What is the level of POS and job performance among administrative staff in Chinese newly-built universities?

Table-1. Mean and standard deviation of POS and job performance

\begin{tabular}{l|l|l|l}
\hline Variables & N & Mean & Std. Deviation \\
\hline POS & 426 & 2.776 & 0.683 \\
\hline Job Performance & 426 & 2.566 & 1.034 \\
\hline
\end{tabular}

As shown in Table 1, both POS and job performance are having their mean below the median of 3 of five-point Likert scale, which is 2.776 and 2.566 respectively. It indicated that the level of POS and job performance among administrative staff in Chinese newly-built universities is slightly low. In other words, administrative staff generally believe that they have not received enough organizational support, and they also feel that they perform not well at work.

Research Question 2: What is the correlation between POS and job performance among administrative staff in Chinese newly-built universities?

Table-2. Correlation analysis between POS and job performance

\begin{tabular}{l|l|l|l}
\hline Variables & N & R-value & P \\
\hline POS & 426 & $0.609 * *$ & 0.000 \\
\cline { 1 - 2 } Job Performance & $* *$ Correlation is significant at the 0.01 level (2-tailed)
\end{tabular}


As presented in Table 2, the value of Pearson correlation between POS and job performance is $0.609(\mathrm{P}<0.01)$. It indicates there is a significant positive correlation between POS and job performance among administrative staff in Chinese newly-built universities.

Research Question 3: Does POS have significant effect on job performance among administrative staff among administrative staff in Chinese newly-built universities?

Table-3. Linear regression of POS on job performance

\begin{tabular}{l|l|l|l|l}
\hline Variables & $\mathbf{N}$ & $\mathbf{R}$ & $\mathbf{R}^{2}$ & $\mathbf{P}$ \\
\hline POS & 426 & 0.609 & 0.371 & 0.000 \\
\hline Dependent variable: Job performance
\end{tabular}

From the result of Table 3, It can be seen that POS has a significant effect on job performance $(\mathrm{R}=0.609$, $\mathrm{P}=0.000$ ). Moreover, the value of $\mathrm{R}$ square of 0.371 indicates that POS explains $37.1 \%$ of the variance in job performance.

\section{Discussion of Findings}

Firstly, the findings showed that administrative staff in Chinese newly-built universities not only have a slightly low level of organizational support, but also have a little bit level of low job performance. It is consistent with the view of some researchers in China who believe that administrative staff is not valued by the university and they perform not well in administration work (Chen, 2017; Yang, 2017). It probably suggested that the government, higher education management authorities and university management levels in China, particularly for newly-built universities, should pay more attention to administrative staff, and find effective ways to improve their POS and job performance.

Secondly, the findings indicated that POS is correlated with job performance positively. This implied that an increase in POS may lead to an increase in job performance. It reminds that POS can be considered as an important factor for improving job performance in higher education management.

Thirdly, the findings revealed that the POS has a significant and positive influence on job performance among administrative staff in Chinese newly-built universities. These findings supported most of the research on POS, they were also consistent with the theory of POS (Afzali et al., 2014; Chen and Yin, 2009; Eisenberger et al., 1986; Mohamed and Ali, 2015; Rhoades and Eisenberger, 2002). This study revealed that the POS theory and the effect of POS on job performance are applicable to most of the organizational culture context. In further, $37.1 \%$ of the variance in job performance is explained by the POS among administrative staff. This suggested that newly-built university can improve the job performance of administrative staff by enhancing their perception of organizational support.

\section{Conclusion and Recommendation}

In accordance with the research findings, the relationship between POS and job performance is positive, and the POS can predict job performance significant among administrative staff of newly-built universities in China. Therefore, it can be recommended for that to elevate the level of job performance of administrative staff in newlybuilt universities, providing more support for them should be emphasized by governments, higher education authorities, policy makers, and university leaders. To be specific, it is suggested to create a comfortable working environment, to provide professional skills training, to recognize and respect administrative staff's value and contribution, to provide opportunities for their career development, to care about their well-being. Certainly, job performance of administrative staff in newly-built universities is a big issue in the context of the popularization of Chinese higher education, which needs more valuable exploration in academic research.

\section{References}

Afzali, A., Motahari, A. A. and Hatami-Shirkouhi, L. (2014). Investigating the influence of perceived organizational support, psychological empowerment and organizational learning on job performance: an empirical investigation. Tehnički Vjesnik, 21(3): 623-29.

Ahmed, I. and Nawaz, M. M. (2015). Antecedents and outcomes of perceived organizational support: a literature survey approach. Journal of Management Development, 34(7): 867-80.

Chen (2017). A study on the job satisfaction of administrative staff in guangdong colleges and universitie (Unpublished master's thesis), (Sun Yat - sen University).

Chen and Yin, X. F. (2009). An empirical study on the relationship between organizational support and job performance in china's real estate industry. Journal of Capital University of Econ. Labrague, L. J., McEnroe Petitte, D. M.

Chiang, C. and Hsieh, T. (2012). The impacts of perceived organizational support and psychological empowerment on job performance: The mediating effects of organizational citizenship behavior. International Journal of Hospitality Management, 31(1): 180-90.

Eisenberger, R., Huntington, R., Hutchison, S. and Sowa, D. (1986). Perceived organizational support. Journal of Applied Psychology, 71(3): 500-07.

Higher Education Evaluation Center of the Chinese Ministry of Education (2017). Chinese Higher Education Quality Report. Beijing. 
Jing, R. T., Gao, X. and Liu, Y. J. (2008). Review on questionnaire survey in China: Situation and suggestion. Management Scientist, (1): 53-57.

Labrague, L. J., McEnroe Petitte, D. M., Leocadio, M. C., Van Bogaert, P. and Tsaras, K. (2018). Perceptions of organizational support and its impact on nurses' job outcomes. Nursing Forum.

Ling, W. Q., Yang, H. J. and Fang, L. L. (2006). Perceived organizational support(POS) of the employees. Journal of Psychological Science, 38(2): 281-87.

Mohamed, S. A. and Ali, M. (2015). The influence of perceived organizational support on employees' job performance. International Journal of Scientific and Research Publication, 5(4): 1-6.

Rhoades, L. and Eisenberger, R. (2002). Perceived organizational support: A review of the literature. Journal of Applied Psychology, 87(4): 698-714.

Yang, H. J. (2017). A study on the relationship between competence and performance of administrative staff in colleges and universitie. Ability and Wisdom, 17(20): 122-23.

$\mathrm{Yu}$, D. C. (1996). The effect of quality factors on job performance of quality managers (Unpublished doctoral thesis), (National Sun Yat - sen University).

Zhang, L. M. and Xie, A. (2015). The strategy of improving the quality of newly-built university administrative staff. Journal of Educational Institute of Jilin Province, 31(12): 8-10.

Zhuang, X. H. (2017). Research on the Construction of Administrative Staff in Newly - built Universities. Theory Research, 58(1): 80-81. 